\title{
Polarization control for fiber systems and devices
}

\author{
D. Tentori, M. A. Cortez Herrera, and A. Rojas-Sánchez \\ Centro de Investigación Cientíica y de Educación Superior de Ensenada, \\ Div. Física Aplicada, Carretera Ensenada-Tijuana No. 3918, Zona Playitas, Ensenada, B.C., 22860 México. \\ e-mail: diana@cicese.mx
}

Received 3 November 2020; accepted 23 November 2020

\begin{abstract}
Nulling the birefringence effect of a light signal traveling through a single-mode fiber when the fiber behaves as an elliptical retarder can be achieved by winding the fiber as a double-helix. We present a resume of the theory and procedures developed at CICESE to demonstrate this polarization control in fiber systems.
\end{abstract}

Keywords: Optical fiber polarization; optical polarization; optical fiber devices.

PACS: 42.81.Gs

\section{Introduction}

Due to the unwanted residual birefringence introduced during the manufacturing process, actual circularly symmetric optical fibers are not perfect. Therefore, they do not maintain the polarization state of the guided light. To reduce the deleterious contributions of light polarization in heterodyne or homodyne optical fiber communications systems [1] and interferometric fiber sensors [2], instead of standard singlemode fibers, polarization-maintaining fibers (PM) or highbirefringence fibers (HiBi) are used. In many of these applications, the output state of polarization (SOP) must remain stable, but PM fibers maintain polarization only when the input polarization is horizontal or vertical. Otherwise, the relative phase of horizontal and vertical components varies in a non-predictable way. Although in HiBi fibers, the coupling between the two modes is much lower, the same limitation is observed. Therefore, sophisticated detection techniques are used. Moreover, since each polarization mode presents a different propagation constant, polarization mode dispersion (PMD) limits their operation bandwidth.

Among the strategies applied to build fiber devices such as lasers, superluminescent light sources, interferometric and polarimetric sensors using single-mode fibers, we can mention the introduction of various polarization controllers $[3,4,5]$. Another alternative has been assuming the fiber exhibits a dominant linear birefringence [6] that can be minimized by applying bending or twist [7]. It was necessary to develop a polarimetric method to characterize a straight untwisted single-mode fiber, to understand birefringence induced by mechanical deformations $[8,9,10]$. Since the reported scientific models did not reproduce the observed experimental results, we evaluated twist-induced birefringence [11]. We found these effects are different for standard and erbium-doped fibers $[12,13,14]$ and determined the methods required to identify residual torsion [15]. These theoretical and experimental results allowed us to analyze the possibility of using a combination of bending and twist (helical winding) to control the output state of polarization emerging from a fiber device $[16,17]$.

\section{Birefringence evaluation of single-mode fibers}

Long-distance communication systems are built with singlemode fibers whose length exceeds several hundreds of kilometers. Therefore, many authors assume that their unwanted birefringence varies randomly. Using PM, HiBi, or standard fibers, since information is encoded using light pulses, birefringence is characterized by polarization mode dispersion or polarization beat length. The proper method for birefringence evaluation depends on the application. For fiber lengths shorter than $100 \mathrm{~m}$, single-mode fiber's birefringence is described using two orthogonal polarization modes whose phase retardation can present a uniform variation [18]. Since the characterization of the ellipticity change of a known state of polarization of light having traveled along the fiber does not supply enough information on the birefringence parameters of the sample [19], researchers have devised several methods based on modulation $[20,21,22]$ and polarimetric techniques [23].

The model we proposed to describe the birefringence of single-mode fibers assumes that absorption is negligible. Under this hypothesis, the Mueller matrices describing birefringence and the Stokes vectors representing the state of light polarization are unitary. Mueller matrice's use simplifies understanding the fiber polarization properties because geometric transformations represent birefringence. A homogeneous retarder model uses a rotation transformation whose axis of rotation intersects the Poincaré sphere at their polarization eigenmodes. The fiber behaves as a linear, circular, or elliptical retarder (general case). 


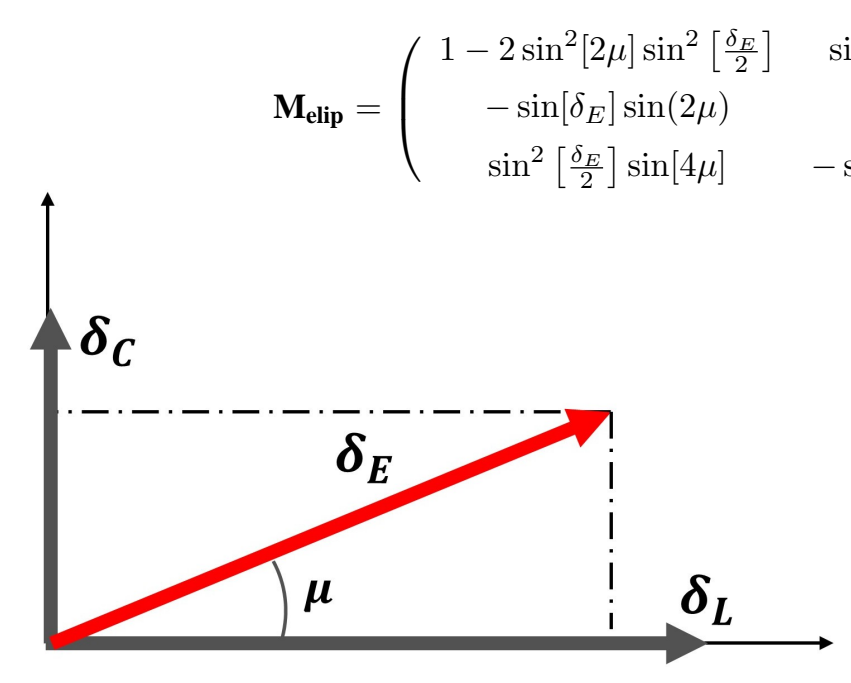

FIGURE 1. An elliptical phase difference between the polarization eigenmodes. It is equal to the geometrical sum of the linear and circular phase differences.

where $\mu$ is the ellipticity angle of the retarder, while the delay angle between the polarization eigenmodes $\delta_{E}$ (elliptical phase difference) is related to the linear and circular phase difference components by $\delta_{E}^{2}=\delta_{L}^{2}+\delta_{c}^{2}$ (Fig. 1).

According to these models, we can follow the evolution of a known input SOP as it propagates through the fiber to identify the type of retarder that describes the fiber birefringence. Although the apparent solution relies on using the cutoff technique, to avoid fiber destruction we can apply wavelength scanning. The method's ability to follow the phase retardation $\left(\delta_{E}\right)$ between the polarization eigenmodes can be understood considering the following relation

$$
\gamma_{E}=\left(\frac{2 \pi}{\lambda}\right) \Delta N L
$$

where $\lambda$ is the signal wavelength, $\Delta N$ the birefringence, and $L$ the fiber length. We obtain a similar behavior for the phase retardation $\delta_{E}$ when the wavelength of a probe signal with a fixed input SOP launched in a fiber with a fixed length is scanned. For simplicity, we selected the known input SOP

$$
\left.\begin{array}{cc}
\sin \left[\delta_{E}\right] \sin [2 \mu] & \sin ^{2}\left[\frac{\delta_{E}}{2}\right] \sin [4 \mu] \\
\cos \left[\delta_{E}\right] & \sin \left[\delta_{E}\right] \cos [2 \mu] \\
\sin \left[\delta_{E}\right] \cos [2 \mu] & 1-2 \cos ^{2}[2 \mu] \sin ^{2}\left[\frac{\delta_{E}}{2}\right]
\end{array}\right),
$$

as linear and considering accuracy, it should be monochromatic [24].

Equation (3) shows the Stokes vector of a linear input SOP with azimuth angle $\phi$ (the superindex $t$ indicates transpose),

$$
\mathbf{S}_{i n}=(\cos [2 \varphi] \sin [2 \varphi] 0)^{t} .
$$

The fiber must be straight and untwisted, keeping the same axial elongation (Fig. 2). We use fiber lengths shorter than $2 \mathrm{~m}$. This limitation comes from the longitudinal span of our optical table.

When a polarized signal is launched at the fiber input and its output state of polarization is measured at the fiber end [Eq. (4)], different traces of the simulated evolution are obtained.

$$
\mathbf{S}_{\text {out }}=\mathbf{M} \mathbf{S}_{\text {in }} .
$$

However, the azimuth angle of the fast birefringence axis of the retarder forms a $\theta$ angle with the measurement reference frame, to compensate for this rotation Eq. (4) is rewritten as

$$
\dot{\mathbf{S}}_{\text {out }}=\mathbf{R}(-\theta) \mathbf{M R}(\theta) \mathbf{S}_{\text {in }},
$$

where $\mathbf{R}$ is a rotation matrix represented mathematically as Eq. (6),

$$
R(\theta)=\left(\begin{array}{ccc}
\cos [2 \theta] & \sin [2 \theta] & 0 \\
-\sin [2 \theta] & \cos [2 \theta] & 0 \\
0 & 0 & 1
\end{array}\right)
$$

Applying Eqs. (3) and (1) in Eq. (5), we obtained the Stokes vector shown in Eq. (7). Mapping the evolution of the output SOP on the Poincaré sphere for different values of the input azimuth angle (10, 20,30, and 40 degrees), we obtained the traces shown in Fig. 3 for an elliptical retarder,

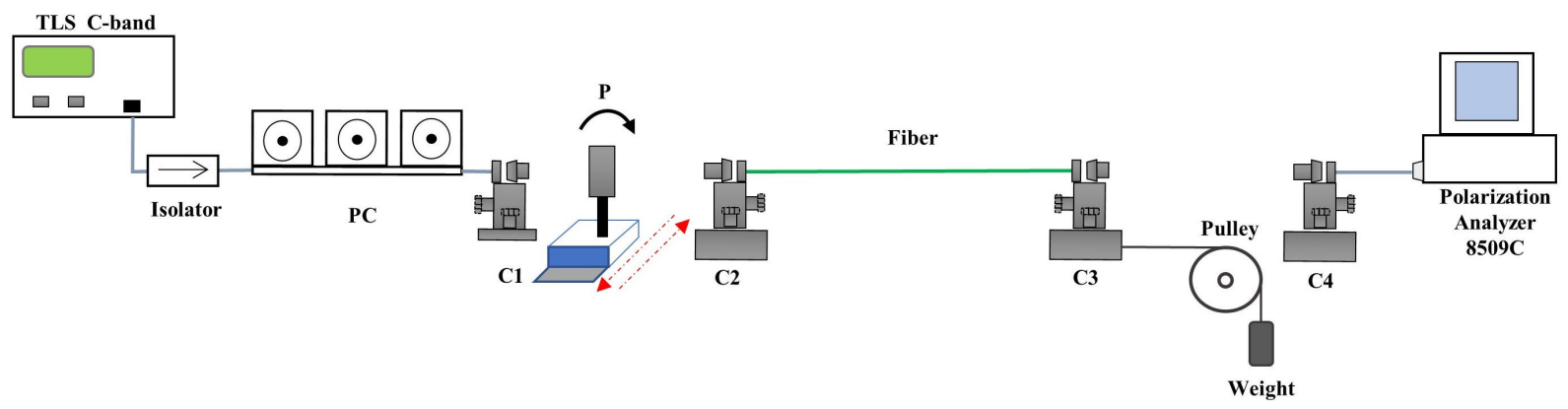

FIGURE 2. A polarimetric set up used to measure the input and output SOPs. The isolator, polarization controller, as well as the input and output couplers $\mathrm{C} 1$ and $\mathrm{C} 4$, remain fixed. To preserve alignment while the reference frame is created for each signal wavelength, the prism polarizer $\mathrm{P}$ and the fiber input coupler $(\mathrm{C} 2)$ are mounted on automatic translation stages. Coupler $\mathrm{C} 3$ is repositioned manually and applies the same elongation to the fiber using the pulley and the weight. 


$$
\left(\begin{array}{c}
\sin [2 \varphi]\left[\cos ^{2}\{2 \mu\} \sin ^{2}\left\{\frac{\delta_{E}}{2}\right\} \sin \{4 \theta\}+\sin \left\{\delta_{E}\right\} \sin \{2 \mu\}\right]+\cos [2 \varphi]\left[\cos \left\{\delta_{E}\right\} \sin ^{2}\{2 \theta\}+\cos ^{2}\{2 \theta\} \varsigma\right] \\
\cos [2 \varphi]\left[\cos ^{2}\{2 \mu\} \sin ^{2}\left\{\frac{\delta_{E}}{2}\right\} \sin \{4 \theta\}-\sin \left\{\delta_{E}\right\} \sin \{2 \mu\}\right]+\sin [2 \varphi]\left[\cos \left\{\delta_{E}\right\} \cos ^{2}\{2 \theta\}+\sin ^{2}\{2 \theta\} \varsigma\right] \\
\cos [2\{\varphi-\theta\}] \sin ^{2}\left[\frac{\delta_{E}}{2}\right] \sin [4 \mu]-\cos [2 \mu] \sin \left[\delta_{E}\right] \sin [2\{\varphi-\theta\}]
\end{array}\right)
$$

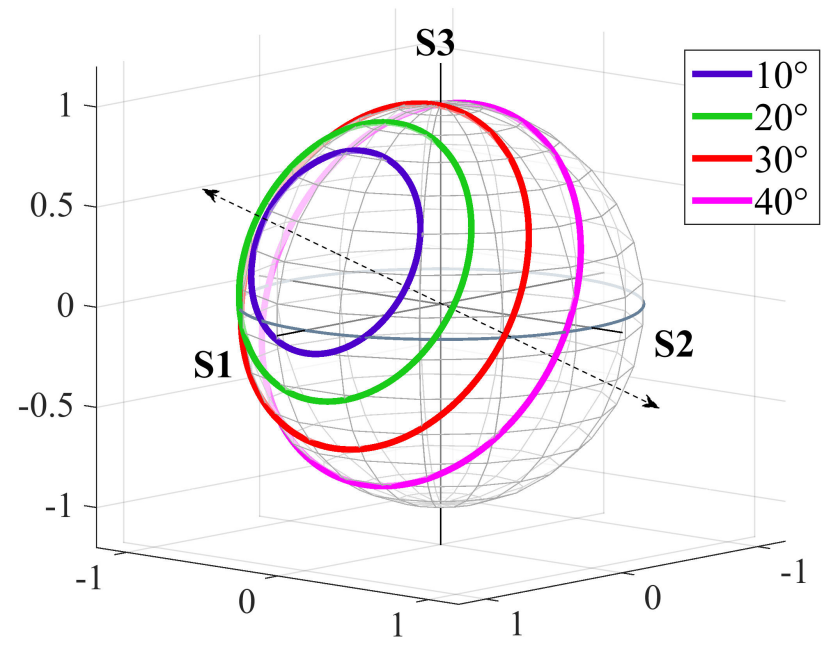

FIGURE 3. SOP evolution along a with fiber whose birefringence corresponds to an elliptical retarder.

where $\varsigma=\left(1-2 \sin ^{2}\left[\delta_{E} / 2\right] \sin ^{2}[2 \mu]\right)$.

Mapping on the Poincaré sphere, the output Stokes parameters measured for the same input linear polarization state (same azimuth angle) allows identifying the type of retarder associated with the fiber's birefringence under evaluation. As an example of these results, see Figs. 6 and 9 in Ref. [9].

After verifying that the fiber behaves as a homogeneous retarder, a linearly polarized monochromatic signal is launched at the fiber input. Varying its azimuth angle from $0^{\circ}$ to $180^{\circ}$, the evolution of the output SOP is measured, and we can determine the ellipticity $(\mu)$, and azimuth $(\theta)$ angles characterizing the polarization eigenmodes, as well as the total retardation angle $\left(\delta_{E}\right)$ modulus- $\pi$. It is essential to emphasize that we use a single azimuth scanning experiment [10].

\section{Twist-induced birefringence}

In an anisotropic medium that twists about the advancing wave's direction, the output SOP rotates with the twist. This behavior is associated with optically active materials; therefore, several authors concluded that torsion induces circular birefringence [7,11]. However, for a fiber that behaves as an elliptical retarder, using the wavelength scanning method, the traces obtained after twisting, shown in Fig. 4, indicate that the fiber no longer behaves as a homogeneous retarder [24].

To understand twist-induced birefringence, we considered that the elastic twisting of fibers in the cold condition causes two effects: a geometrical rotation of the fiber's birefringence axes with the twist rate, and torsional stress. Assuming the fiber is formed by thin slices with a plane and parallel faces and using matrix calculus, we have that the total birefringence of the fiber is given by [12].

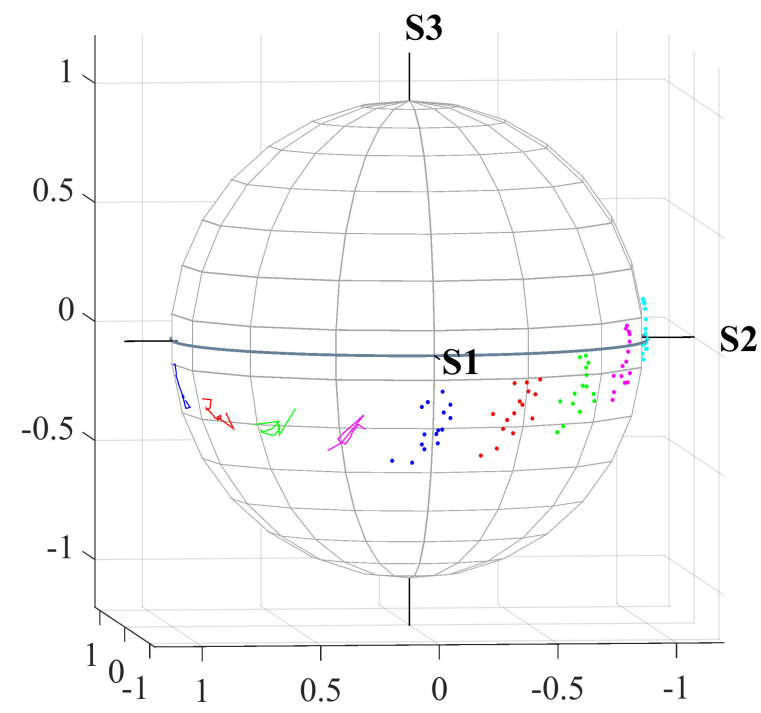

FIGURE 4. Evolution of the state of polarization of a twisted fiber $(58.5 \mathrm{rad} / \mathrm{m})$ determined using the wavelength scanning method. Traces were elaborated for SOP azimuth angles from $0^{\circ}$ to $80^{\circ}\left(10^{\circ}\right.$ increment $)$. Each trace was elaborated using wavelengths between 1517 and $1569 \mathrm{~nm}$.

$$
\mathbf{M}_{g \tau}=\mathbf{R}(\tau) \mathbf{M}\left(\delta_{E}\right),
$$

where $\tau$ is the total angle of twist and $\mathbf{M}\left(\delta_{E}\right)$ is the original fiber birefringence.

Working within the elastic limit, for standard fibers, the output SOP of the signal presents a variation of the Stokes parameters similar to that shown in Fig. 5a). We can see that the Stokes parameter S3 oscillates, keeping a constant amplitude, around a fixed horizontal line. We have shown that in this case, the birefringence matrix is [13]

$$
\begin{aligned}
\mathbf{M}\left(\tau, \delta_{E}(\tau), \theta, \mu\right)=\mathbf{R}(-\theta) \\
\times\left(\mathbf{R}[b \tau+\beta] \mathbf{M}_{\tau}\left[\delta_{E}\{\tau\}, \mu\right]\right) \mathbf{R}(\theta),
\end{aligned}
$$

where $R(\theta)$ and $R(-\theta)$ describe the orientation of the fiber birefringence matrix with respect to the reference system of the laboratory, $R(b \tau)$ corresponds to the geometric rotation $R(\tau)$ of Eq. 8, $\beta$ is a fixed rotation angle used to match the theoretical reference frame with that of the polarization analyzer. Here $b$ is a constant for each fiber sample (not depending on the wavelength) whose value is close to 1 . The ellipticity angle $\mu$ was constant for this standard fiber. After analyzing several options, we proposed that phase retardation $\delta_{E}$ varies linearly with the applied twist

$$
\delta_{E}(\tau)=\delta_{E}(0)+c \tau,
$$




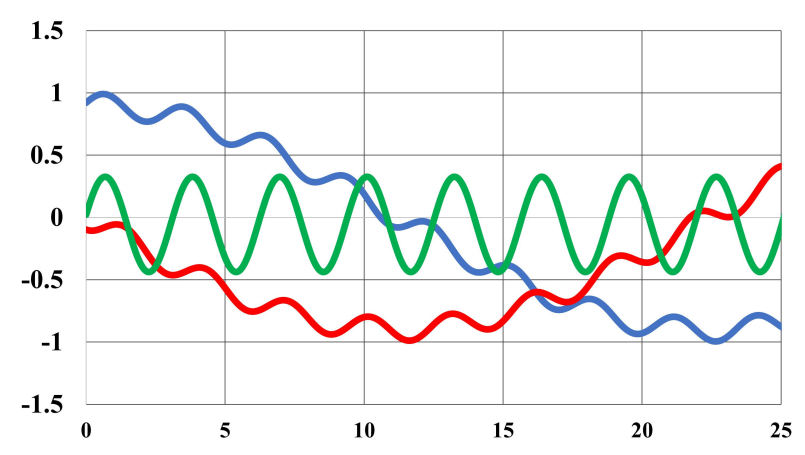

a) $-\mathrm{S} 1-\mathrm{S} 2-\mathrm{S3}$

1.5

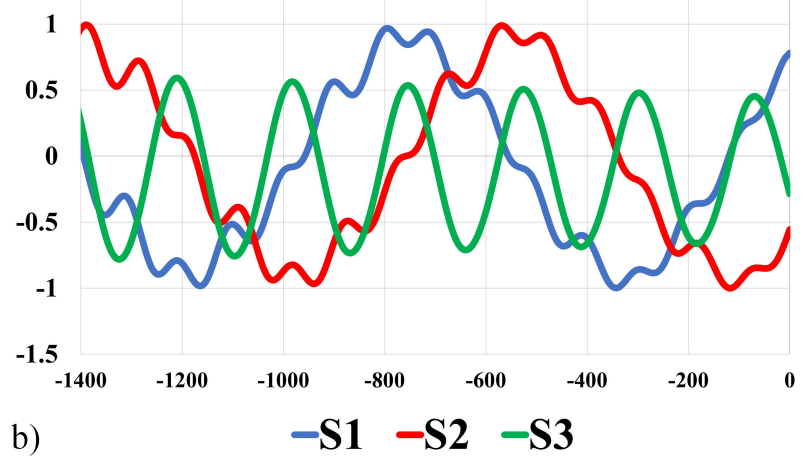

FIgURE 5. Output Stokes parameters measured for a) a standard fiber (SMF28) varying the twist angle from 0 to $25 \mathrm{rad}\left(0^{\circ}\right.$ to $1432^{\circ}$ ). S3 oscillates around a horizontal line keeping a fixed amplitude, b) an erbium-doped fiber (INO 402K5) varying the twist angle from $0^{\circ}$ to $1400^{\circ}$. S3 oscillates with a non-fixed amplitude around an inclined line.

where $c$ is a constant for each fiber sample, not depending on the wavelength. The numerical simulation allows the determination of its value from experimental data.

Twisting an erbium-doped fiber, the results obtained for the output Stokes parameters present a different behavior. In this case, parameter $S_{3}$ oscillates around an inclined line, and its oscillation amplitude is not constant. To understand the relations between the parameters describing the twisted fiber's birefringence, we used the differential description of birefringence matrices developed by R. C. Jones [25]. We obtained a more robust modification of polarization properties in erbium-doped fibers (EDFs) than in undoped single-mode fibers. Its description requires a model that, in addition to a linear component at $0^{\circ}$, includes a linear birefringence component at $45^{\circ}$ (Jones birefringence) and a circular component. All these birefringence components are twist dependent.

We should mention that for erbium-doped fibers behaving as homogeneous retarders, ellipticity and azimuth birefringence components present spectral behaviors that resemble the EDFs fluorescence emission curve [9]. Since KramersKrönig relations tie the frequency response of the material's absorption coefficient to its refractive index chromatic dispersion, we hypothesize this behavior might be linked to absorption. Further work must verify if it presents the same performance for different gain media (Ho, Tm, Yb).

We found that most fibers exhibit a different response to right- and left-handed twists. This behavior, explained for standard fibers [15], was also observed for EDFs. We should mention that a procedure for the identification of residual torsion using a null polarimeter and the Jones formalism has been reported in Sec. 2.2.2 of Ref. [26]. Regarding the wavelength scanning technique, it was reported for a Corning EDF (Fig. 4c, Ref. [9]), and also corresponds to the SOP evolution traces shown in Fig. 4 for a twisted fiber.

\section{Polarization control using helices}

Using single-mode fibers in the construction of some fiber systems, residual birefringence has led the designers to use polarization controllers (PC) to adjust the light signal's state of polarization. However, PCs are built with three nonregular fiber windings. Thus its output polarization cannot be predicted. To optimize polarization control, two or more PCs are used and adjusted by trial and error. In what follows, we describe a more precise alternative to control the polarization performance of fiber systems and devices based on the use of helically wound fibers.

The polarization properties of a helical coil include a geometrical contribution due to the out-of-plane trajectory described by the light beam traveling along with the fiber and bend- and twist-induced birefringence. About the topological contribution introduced by the helical trajectory followed by light along with the fiber, if the photoelastic contribution is neglected, it is described as [27]

$$
\mathbf{M}_{H}=\mathbf{R}(\Omega) \mathbf{M}
$$

where, $\Omega=n 2 \pi(1+\sin \xi)$; here $n$ is the number of full turns around the helix axis, and $\xi$ is the helix pitch angle. We can see that equal and opposite rotations are canceled out. Hence the topological rotation of a double helix with equal but opposite gyrations is null.

On the one hand, since the birefringence resulting from bending is linear [28], for an optical fiber behaving as an elliptical retarder, we can see from Fig. 1 that by adding a linear birefringence component, the new elliptic retarder will exhibit higher linear retardation. On the other hand, regarding torsion, the twist-induced birefringence of an elliptical retarder is described with Eq. (9). Furthermore, when helically induced birefringence is the dominant effect, the curvature's normal vector defines the fiber's fast birefringence axis orientation [29]. Therefore, aligning the helices with the fiber system is easy.

We showed that for two identical helical windings with opposite handedness, built with standard optical fiber, the birefringence can be canceled out [17]. Therefore, a double helix can control the polarization of the light beam traveling along with the fiber system. If required, this polarization control can be accomplished at each system's components and the sensing or gain fiber. The experimental methodology used to build these double helices has been reported in Ref. [17]. 
Based on our ability to control the effect of fiber birefringence on the output state of polarization, we currently study the physical phenomena involved in the fluorescent emission of superluminescent fiber sources and the polarizationdependent gain in erbium-doped fiber amplifiers.

\section{Conclusion}

In this work, we present a method allowing the control of the polarization performance of fiber systems. Since the control is based on the use of two helical windings, to demonstrate its ability for this purpose, it was necessary to obtain the birefringence matrix of a helical coil.
A helical coil presents two mechanical deformations (bending and twist) and a topological contribution. We mentioned previous results showing that for helices with opposite winding, the topological and the photoelastic contributions can be canceled out. We cited the birefringence's mathematical description induced by a helical coil, which we deduced from the results reported for bend- and twist-induced birefringence. We developed the matrix description of twistinduced birefringence. To apply the matrix model developed for twist-induced birefringence, we must verify the birefringence of the fibers that will be used corresponds to that of an elliptical retarder. Therefore we presented the methods we developed to perform this characterization.
1. T. Okoshi, Polarization-state control schemes for heterodyne or homodyne optical fiber communications, IEEE Transactions on Electron Devices, 32 (1985) 2624. https : / / doi .org/10. $1109 / \mathrm{T}-\mathrm{ED} .1985 .22393$

2. D. Leandro, M. Bravo, A. Ortigosa, M. Lopez-Amo, RealTime FFT Analysis for Interferometric Sensors Multiplexing, J. Lightwave Technol 33 (2015) 354. https://doi.org/ 10.1109 /JLT.2014.2388134

3. Dakin, John; Culshaw, Brian, Optical fiber sensors: Principles and components. Vol. 1 (Boston, MA, Artech House, 1988), p. 343.

4. F. Amrani, M. Salhi, P. Grelu, H. Leblond, and F. Sanchez, Universal soliton pattern formations in passively mode-locked fiber lasers, Opt. Lett. 36 (2011) 1545. https://doi.org/10. $1364 /$ OL.36.001545

5. Hee Gap Park, Michel Digonnet, and Gordon Kino, ErDoped Superfluorescent Fiber Source With a \pm 0.5 - ppm LongTerm Mean-Wavelength Stability, J. Lightwave Technol. 21 (2003) 3427. https://doi.org/10.1109/JLT.2003. 822539

6. I. Kaminow, Polarization in optical fibers, IEEE Journal of Quantum Electronics 17 (1981) 15. https://doi.org/ 10.1109/JQE.1981.1070626

7. A. M. Smith, Birefringence induced by bends and twists in single-mode optical fiber, Appl. Opt. 19 (1980) 2606. https : //doi.org/10.1364/AO.19.002606

8. F. Treviño-Martínez, D.Tentori, C.Ayala-Díaz, F. MendietaJiménez, Birefringence assessment of single-mode optical fibers, Opt. Express 13 (2005) 2556. https://doi.org/ 10.1364 /OPEX.13.002556.

9. D. Tentori, C. Ayala-Díaz, F. Treviño-Martínez, F.J. MendietaJiménez, Evaluation of the residual birefringence of singlemode erbium-doped silica fibers, Opt Commun 271 (2007) 73. https://doi.org/10.1016/j.optcom.2006.09. 068

10. A. Rojas-Sanchez and D. Tentori, Simple method for the characterization of birefringence of single-mode optical fibers, $O S A$ Continuum 3 (2020) 1650. https: //doi.org/10.1364/ OSAC. 383249
11. R. Ulrich and A. Simon, Polarization optics of twisted singlemode fibers, Appl. Opt. 18 (1979) 2241. https://doi. org/10.1364/AO.18.002241

12. D. Tentori, C. Ayala-Díaz, E. Ledezma-Sillas, F. TreviñoMartínez, A. García-Weidner, Birefringence Matrix for a Twisted Single-Mode Fiber: Geometric Contribution, Opt. Commun. 282 (2009) 830. https: //doi.org/10.1016/ j.optcom.2008.11.011

13. D. Tentori, C. Ayala-Díaz, A. García-Weidner, Birefringence Matrix for a Twisted Single-Mode Fiber: Photoelastic and Geometrical Contributions, Optical Fiber Technology, 18 (2012) 14. https://doi.org/10.1016/j.yofte. 2011.10 .001

14. Diana Tentori, A. Garcia-Weidner, Jones birefringence in twisted single-mode optical fibers, Opt. Express 21 (2013) 31725. https://doi.org/10.1364/OE.21.031725

15. D. Tentori, A. García-Weidner, Right- and left-handed twist in optical fibers, Rev. Mex. Fis. 60 (2014) 69.

16. D. Tentori, A. García Weidner y Ernesto Ledezma Sillas, Birefringence description of a helical fiber-coil, Rev. Mex. Fis. 62 (2016) 489.

17. D. Tentori, A. García-Weidner y Jorge Arturo Rodríguez García, Use of fiber helical coils to obtain polarization insensitive fiber devices, Opt Fiber Technol, 31 (2016) 13. https: //doi.org/10.1016/j.yofte.2016.05.002

18. B. Ibarra-Escamilla, E.A. Kuzin, F. Gutierrez-Zainos, R. Tellez-Garcia, J.W. Haus, R. Rojas-Laguna, J.M. EstudilloAyala, Measurement of beat length in short low-birefringence fibers using the fiber optical loop mirror, Opt Commun 217 (2003) 211. https://doi.org/10.1016/ S0030-4018(03) 01117-9

19. V. Ramaswamy, R.D.Standley, D.Sze, W.G.French, Polarization effects in short length, single mode fibers, Bell Sys. Tech. J. 57 (1978) 635. https://doi.org/10.1002/j. 1538-7305.1978.tb00599.x

20. K. Takada, J. Noda, and R. Ulrich, Precision measurement of modal birefringence of highly birefringent fibers by periodic lateral force, Appl. Opt. 24 (1985) 4387. https : / / doi. org/10.1364/AO.24.004387 
21. A. Barlow, Optical-fiber birefringence measurement using a photo-elastic modulator, J. Lightwave Technology, 3 (1985) 135. https://doi.org/10.1109/JLT.1985. 1074139

22. Chang-Seok Kim, Young-Geun Han, R. M. Sova, Un-Chul Paek, Youngjoo Chung and J. U. Kang, Optical fiber modal birefringence measurement based on Lyot-Sagnac interferometer, IEEE Photonics Techn. Lett., 15 (2003) 269. https: //doi.org/10.1109/LPT.2002.806892

23. R. Calvani, R. Caponi and F. Cisternino, Polarization measurements on single-mode fibers, J. Lightwave Technol 7 (1989) 1187.https://doi.org/10.1109/50.32381

24. D. Tentori, C. Ayala-Díaz, F. Treviño-Martínez, Twist-induced birefringence in fibers and optical rotation, Proc. SPIE 7499 (2009) 74991010. https://doi.org/10.1117/ 12.843817
25. R. C. Jones, A new calculus for the treatment of optical systems. VII. Properties of the N-matrices, J. Opt. Soc. Am. 38 (1948) 671. https: //doi.org/10.1364/JOSA. 38.000671 .

26. D. Tentori, A. Garcia-Weidner and Evgeni Kuzin, On the birefringence evaluation of single-mode fibers, Rev. Mex. Fis. 62 (2016) 381

27. D. Tentori, C. Ayala-Díaz, F. Treviño-Martínez, F.J. MendietaJiménez, H. Soto-Ortiz, Birefringence evaluation of helically wound optical fibres, J. Modern Opt 48 (2001) 1767 https : //doi.org/10.1080/09500340108231432

28. R. Ulrich, S. C. Rashleigh, and W. Eickhoff, Bending-induced birefringence in single-mode fibers, Opt. Lett. 5 (1980) 273 https://doi.org/10.1364/OL.5.000273 